\title{
Experience of percutaneous tracheostomy in critically ill COVID-19 patients
}

\author{
Eun Jin Kim ${ }^{1, *}$, Eun-Hyung Yoo ${ }^{2 *}$, Chi Young Jung ${ }^{1}$, Kyung Chan Kim ${ }^{1}$ \\ Departments of ${ }^{1}$ Internal Medicine and ${ }^{2}$ Laboratory Medicine, Daegu Catholic University Medical Center, Daegu Catholic University School of Medicine, \\ Daegu, Korea
}

Background: Coronavirus disease 2019 (COVID-19) is a highly contagious disease that causes respiratory failure. Tracheostomy is an essential procedure in critically ill COVID-19 patients; however, it is an aerosol-generating technique and thus carries the risk of infection transmission. We report our experience with percutaneous tracheostomy and its safety in a real medical setting.

Methods: During the COVID-19 outbreak, 13 critically ill patients were admitted to the intensive care unit (ICU) at Daegu Catholic University Medical Center between February 24 and April 30, 2020. Seven of these patients underwent percutaneous tracheostomy using Ciaglia Blue Rhino. The medical environment, percutaneous tracheostomy method, and COVID-19 reverse transcriptase-polymerase chain reaction (RT-PCR) results were retrospectively reviewed. After treatment, the COVID-19 infection status of healthcare personnel was investigated by RT-PCR.

Results: The ICU contained negative pressure cohort areas and isolation rooms, and healthcare personnel wore a powered air-purifying respirator system. We performed seven cases of percutaneous tracheostomy in the same way as in patients without COVID-19. Five patients (71.4\%) tested positive for COVID-19 by RT-PCR at the time of tracheostomy. The median cycle threshold value for the RNA-dependent RNA polymerase was 30.60 (interquartile range [IQR], 25.50-36.56) in the upper respiratory tract and $35.04(I O R, 28.40-36.74)$ in the lower respiratory tract. All healthcare personnel tested negative for COVID-19 by RT-PCR.

Conclusions: Percutaneous tracheostomy was performed with conventional methods in the negative pressure cohort area. It was safe to perform percutaneous tracheostomy in an environment of COVID-19 infection.

Key Words: COVID-19; reverse transcriptase polymerase chain reaction; SARS-CoV-2; tracheostomy

\section{INTRODUCTION}

Coronavirus disease 2019 (COVID-19) caused by severe acute respiratory syndrome coronavirus 2 (SARS-CoV-2) has spread rapidly around the world since it was first reported in Wuhan, China in December 2019 [1]. People infected with SARS-CoV-2 display various clinical symptoms, ranging from asymptomatic to acute respiratory distress syndrome (ARDS). COVID-19 is reported to cause ARDS in 15.6\%-31\% of cases [1-6]. SARS-CoV-2 seems to have a lower mortality than SARS and Middle East respiratory syndrome (MERS); however, its infec-

\section{Original Article}

Received: June 25, 2020

Revised: September 14, 2020

Accepted: September 29, 292020

\section{Corresponding author}

Eun Jin Kim

Department of Internal Medicine,

Daegu Catholic University

Medical Center, Daegu Catholic

University School of Medicine, 33

Duryugongwon-ro 17-gil, Nam-gu,

Daegu 42472, Korea

Tel: +82-53-650-4274

Fax: +82-53-650-4942

E-mail: ejkim77@cu.ac.kr

*These authors contributed equally to this article.

Copyright $\odot 2020$ The Korean Society of Critical Care Medicine

This is an Open Access article distributed under the terms of Creative Attributions Non-Commercial License (https:// creativecommons.org/li-censes/by-nc/4.0/) which permits unrestricted noncommercial use, distribution, and reproduction in any medium, provided the original work is properly cited. 
tivity is very high [7].

Tracheostomy is a common procedure in patients who require prolonged ventilation. It can facilitate weaning from a ventilator and improve availability in intensive care units (ICUs) [8]. However, tracheostomy is an aerosol-generating procedure and thus carries the risk of infecting healthcare personnel with SARS-CoV-2. There were several case reports of tracheostomy during the SARS [9-11] and MERS outbreaks [12], all of which were surgical tracheostomy. Surgical tracheostomies were generally favored over percutaneous tracheostomies [8]. In SARS-CoV-2 infection, several guidelines have been published to reduce the risk of infection [8,13-15]. Due to the sudden increase in critically ill patients with SARS-CoV-2 infection in South Korea, we created a temporary airborne infection isolation area with negative pressure in our ICU environment where percutaneous tracheostomy was performed. We report our experiences with percutaneous tracheostomy in a medical environment and factors related to infection of healthcare personnel.

\section{MATERIALS AND METHODS}

During the COVID-19 outbreak in South Korea, 133 COVID-19 patients were admitted to Daegu Catholic University Medical Center between February 24 and April 30, 2020, of whom 13 were admitted to the ICU. Ten of these 13 patients required ventilators, of whom seven underwent percutaneous tracheostomy. Patient basic clinical characteristics, the medical environment at the time of tracheostomy, ventilator-related settings, the percutaneous tracheostomy method, and SARS$\mathrm{CoV}-2$-related information were retrospectively reviewed. After treatment, real-time reverse transcriptase-polymerase chain reaction (RT-PCR) was used to investigate whether healthcare personnel who had been working at the ICU were infected with SARS-CoV-2.

This study was reviewed and approved by the Institutional Review Board of Daegu Catholic University Medical Center (IRB No. CR-20-083). The requirement for informed consent was waived because of the retrospective study design.

\section{Environments}

After the first case of COVID-19 was confirmed on February 18, 2020 in the Daegu region, the number of COVID-19 patients requiring intensive care began to increase. Like other hospitals in the same area [16], our hospital created a temporary airborne infection isolation area with negative pressure to care for critically ill patients with COVID-19. The unit con-

\section{KEY MESSAGES}

- It is safe to perform percutaneous tracheostomy in an environment where patients have coronavirus disease 2019 (COVID-19).

- The risk of infection transmission associated with tracheostomy is thought to be reduced by use of personal protective equipment, a negative pressure environment, and in the presence of a low viral load.

sisted of nine beds; three beds were in negative pressure isolation rooms with one anteroom, and six beds were in cohort areas that used a common space and a mobile negative air machine to create negative pressure. The entire unit was sealed by erecting a temporary wall to maintain proper negative pressure, and all spaces were subjected to pressure monitoring (Figure 1). Medical personnel used the 3M Jupiter powered air-purifying respirator system (PAPR), a 3M Versaflo high durability hood with integrated head suspension, eye protection, a fluid-repellent disposable surgical gown, a cap, boots, double gloves, and an N95 mask (Figure 2).

\section{Procedures}

This hospital has a protocol to perform a tracheostomy after about 14 days for patients who are on a ventilator. Percutaneous tracheostomy was performed at the bedside by an experienced physician who has worked in the ICU for 4 years and has performed more than 100 such procedures. A sealed ventilator circuit was used as the ventilator. A single-use disposable flexible bronchoscope (Ambu aScope 3 Broncho Large 5.8/2.8; Ballerup, Denmark) was employed. Ambu aView was used as the bronchoscopic monitor. One operator and one respiratory specialist examining the bronchoscopy were in close proximity to the patient. One nurse assisted with the procedure. The resident doctor also helped in some cases (Figure 2). Tracheostomy was performed using the Blue Rhino single dilation technique [17] and Ciaglia Blue Rhino (Cook Critical Care, Bloomington, IN, USA).

All patients received general intravascular anesthesia consisting of dexmedetomidine, remifentanil, and midazolam. Neuromuscular blockade was achieved using cisatracurium, which was titrated to prevent spontaneous respiration, cough, and physical movement. All patients received volume-controlled ventilation of the lungs with a fraction of inspired oxygen $\left(\mathrm{FiO}_{2}\right)$ of $100 \%$ during the procedure. The positive end-expiratory pressure (PEEP), inspiratory pressure, respiratory rate, and inspiration:expiration ratio were maintained at the 


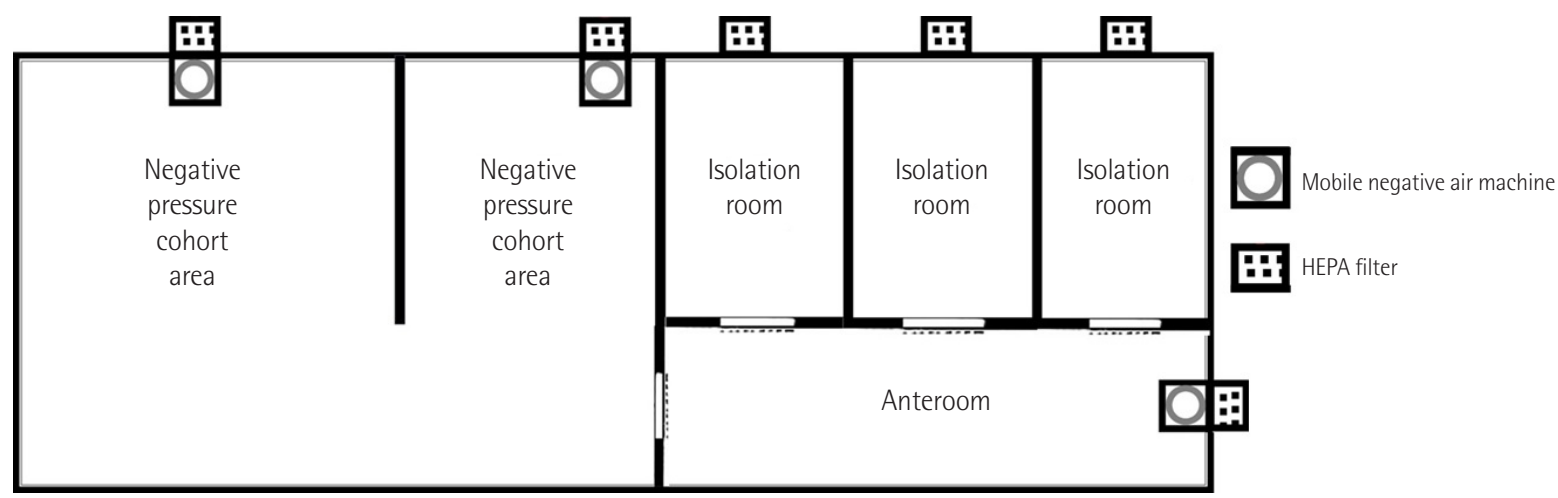

Figure 1. Schematic view of the temporary negative pressure isolation intensive care unit. The unit consisted of nine beds; three beds were in negative pressure isolation rooms with one anteroom, and six beds were in cohort areas that used a common space and a mobile negative air machine to create negative pressure. The entire unit was sealed by erecting a temporary wall to maintain proper negative pressure, and all spaces were subjected to pressure monitoring.

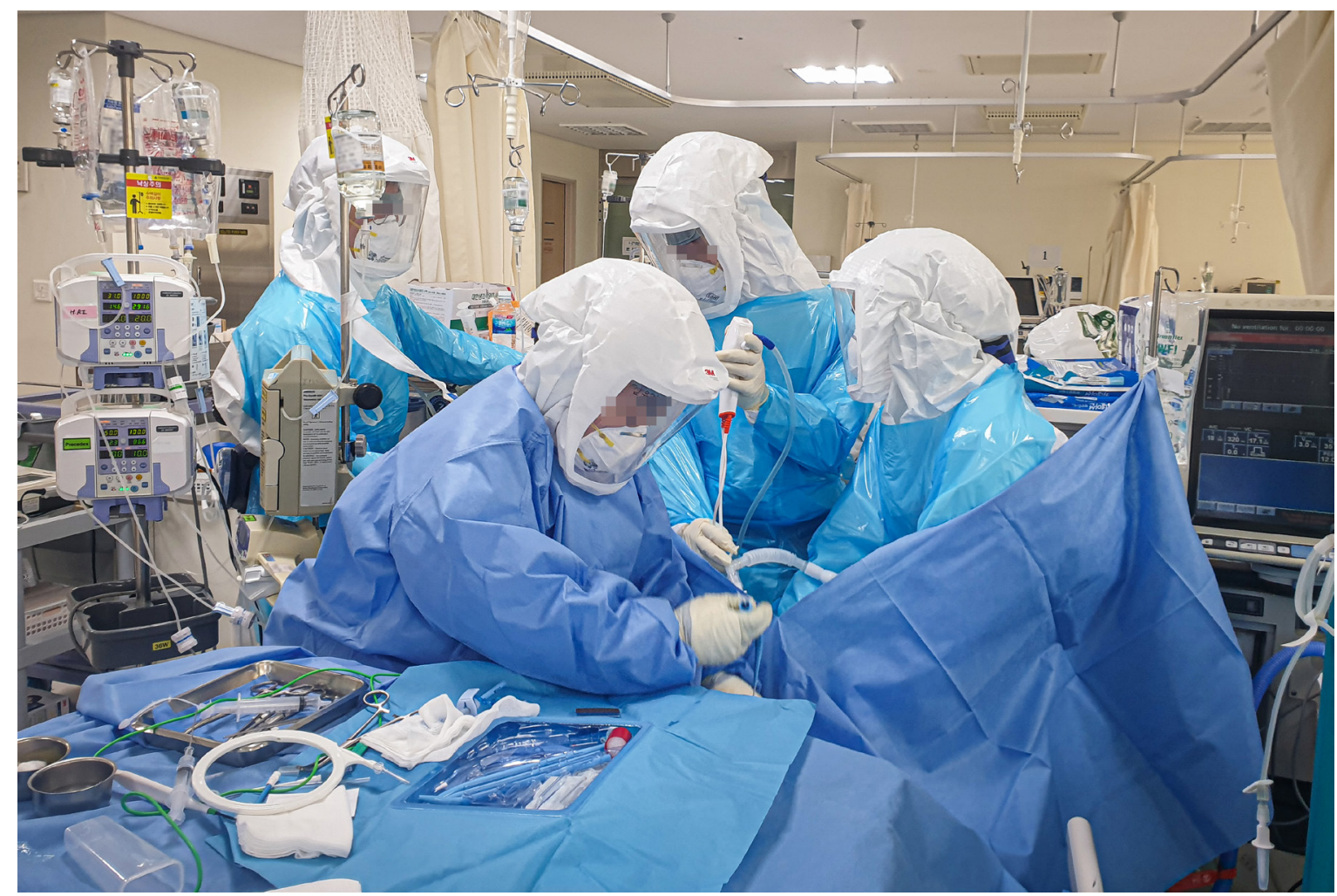

Figure 2. A photograph of healthcare personnel performing percutaneous tracheostomy. Healthcare personnel wore a 3M Jupiter powered air-purifying respirator system, a 3M Versaflo high durability hood with integrated head suspension, eye protection, a fluid-repellent disposable surgical gown, a cap, boots, double gloves, and an N95 mask.

same levels as those used prior to the procedure. The patient was in the supine position and his/her neck was extended backward. The operator checked the blood vessels and structures on the patient's anterior neck by ultrasonography and identified areas that were considered safe, usually the first two or three tracheal rings. Lidocaine was injected into the intended tracheostomy site and a skin incision of $2 \mathrm{~cm}$ or less was made. Pretracheal tissues were bluntly dissected using electrocauterization to expose the pretracheal fascia. The bronchoscope was placed in the endotracheal tube (ETT), drawn backwards from the ETT, and positioned above the intended puncture site. The trachea was punctured with a 14-gauge cannula-on-needle in a posterio-caudad direction. Tracheal entry of the needle was confirmed by aspiration of air into a 
saline-filled syringe.

After successful puncture, a guidewire was passed through the cannula into the tracheal lumen. The needle and needle cannula were then withdrawn. A 14-French short introducer dilator was then passed over the guidewire and subsequently removed. A lubricated Ciaglia Blue Rhino dilator containing the guiding catheter was passed over the guidewire. The dilator was then removed while the guiding catheter remained. The tracheostomy tube that was loaded over an appropriate introducer was inserted through the tracheal stoma over the guidewire and guiding catheter. The introducer, guidewire, and guiding catheter were then removed, leaving the tracheostomy tube in situ. During insertion and removal of catheters, guidewires, and dilators, the stoma site was covered with gauze and the operator's hands. Bronchoscopy was performed throughout the procedure to ensure correct tracheal placement of the needle, guidewire, and dilator, and final insertion of the tracheostomy tube. Tracheostomy-related complications, procedure time, ventilator settings, and laboratory test results at the time of tracheostomy were investigated. Procedure time was defined as the duration from neck incision to tracheostomy tube insertion. The ventilator was not paused while the tracheostomy tube was inserted.

\section{Detection of SARS-CoV-2}

SARS-CoV-2 was detected by real-time RT-PCR. RNA was extracted from clinical samples using a Nextractor NX-48 kit (Biosewoom, Seoul, Korea) following the manufacturer's instructions. Real-time RT-PCR to detect SARS-CoV-2 was performed using a PowerchekTM 2019-nCoV Real-time PCR kit (Kogenebiotech, Seoul, Korea). Thermal cycling was performed at $50^{\circ} \mathrm{C}$ for 30 minutes for reverse transcription, followed by inactivation of the reverse transcriptase at $95^{\circ} \mathrm{C}$ for 10 minutes. PCR amplification was performed with 40 cycles of $95^{\circ} \mathrm{C}$ for $15 \mathrm{sec}-$ onds and $60^{\circ} \mathrm{C}$ for 1 minute in the CFX 96TM real-time PCR system (Bio-Rad, Hercules, CA, USA). All specimens were handled in a biosafety cabinet. RT-PCR testing was performed according to the proposed guidelines for diagnosing COVID-19 in clinical laboratories issued by the Korea Centers for Disease Control and Prevention and the Korean Society for Laboratory Medicine. Cycle threshold (Ct) values were determined. High Ct values for the RNA-dependent RNA polymerase (RdRp) and $\mathrm{E}$ (envelop) genes were interpreted as negative results. The results of quantitative RT-PCR were collected for viral load quantification. Ct values close to the cutoff in specimens with low viral loads were interpreted by a laboratory physician, and retests were performed using residual or new specimens if nec- essary. We assessed anti-SARS-CoV-2 immunoglobulin G (IgM) and IgG antibodies using the DIAKEY COVID-19 IgM/IgG rapid kit (Shin Jin Medics, Goyang, Korea).

\section{Statistical Analysis}

Test results are presented as absolute values and percentages. Continuous variables were not normally distributed and therefore medians and interquartile ranges (IQRs) are presented. Statistical analysis was performed using IBM SPSS ver. 25.0 (IBM Corp., Armonk, NY, USA).

\section{RESULTS}

Between February 24 and April 30, 2020, 13 patients were admitted to the ICU. Ten of these 13 critically ill patients required ventilators, of whom seven underwent percutaneous tracheostomy and three patients had ventilators removed without tracheostomy. Seven critically ill COVID-19 patients underwent percutaneous tracheostomy. The median age was 71 years (IQR, 63-76 years) and the male:female ratio was 6:1. The median body mass index was $27.2 \mathrm{~kg} / \mathrm{m}^{2}$ (IQR, 22.8-28.2 $\mathrm{kg} / \mathrm{m}^{2}$ ). The median duration from symptom onset to diagnosis was 2 days (IQR, $0-4$ days), the median duration from symptom onset to application of the ventilator was 7 days (IQR, 3-12 days), and the median duration from application of the ventilator to percutaneous tracheostomy was 14 days (IQR, 9-16 days). The median Acute Physiology and Chronic Health Evaluation II (APACHE II) score was 15 (IQR, 12-17). Five cases (71.4\%) were previous or current smokers (Table 1).

The median operating time for percutaneous tracheostomy was 10 minutes (IQR 7-12 minutes). The ventilator settings during percutaneous tracheostomy were as follows: median PEEP, $12 \mathrm{~cm} \mathrm{H}_{2} \mathrm{O}$ (IQR, 10-14 cm $\mathrm{H}_{2} \mathrm{O}$ ) and median $\mathrm{FiO}_{2}$, 0.6 (IQR, 0.35-0.80). Extracorporeal membrane oxygenation (ECMO) was used in two cases (28.6\%) and continuous renal replacement therapy was used in three cases $(42.9 \%)$. $\mathrm{FiO}_{2}$ was 0.35 when ECMO was used. The median platelet count was $159 \times 10^{3} / \mu \mathrm{l}\left(\mathrm{IQR}, 82-196 \times 10^{3} / \mu \mathrm{l}\right.$; normal range, $\left.140-380 \times 10^{3} / \mu \mathrm{l}\right)$, the median prothrombin time was 14.6 seconds (IQR, 13.915.4 seconds; normal range, 11.5-15.0 seconds), the median prothrombin time and international normalized ratio was 1.17 (IQR, 1.06-1.25; normal range, 0.8-1.2), and the median Activated Partial Thromboplastin Time was 37.5 seconds (IQR, 36.2-46.4 seconds; normal range, 28.0-45.0 seconds).

Postoperative bleeding was the only complication after tracheostomy. This occurred in two patients (28.6\%), both of whom were on ECMO support and anticoagulation therapy. Of them, 
Table 1. Basic characteristics and tracheostomy-related outcomes in the study population

\begin{tabular}{|c|c|}
\hline Variable & Value $(n=7)$ \\
\hline Age (yr) & $71(63-76)$ \\
\hline Male:female & $6: 1$ \\
\hline Body mass index $\left(\mathrm{kg} / \mathrm{m}^{2}\right)$ & $27.2(22.8-28.2)$ \\
\hline APACHE II score & $15(12-17)$ \\
\hline \multicolumn{2}{|l|}{ Smoking status } \\
\hline Never & $2(28.6)$ \\
\hline Previous smoker & $4(57.1)$ \\
\hline Current smoker & $1(14.3)$ \\
\hline \multicolumn{2}{|l|}{ Comorbidity } \\
\hline Diabetes mellitus & $3(42.9)$ \\
\hline Hypertension & $2(28.6)$ \\
\hline Chronic lung disease & $2(28.6)$ \\
\hline Malignancy, solid & $1(14.3)$ \\
\hline $\begin{array}{l}\text { Time from symptom onset to application of the } \\
\text { ventilator (day) }\end{array}$ & $7(3-12)$ \\
\hline $\begin{array}{l}\text { Time from symptom onset to percutaneous } \\
\text { tracheostomy (day) }\end{array}$ & $20(17-24)$ \\
\hline $\begin{array}{l}\text { Time from application of the ventilator to } \\
\text { percutaneous tracheostomy (day) }\end{array}$ & $14(9-16)$ \\
\hline Procedure time of percutaneous tracheostomy ${ }^{a}(\mathrm{~min})$ & $10(7-12)$ \\
\hline Tracheostomy tube size, internal diameter (mm) & 8 \\
\hline \multicolumn{2}{|l|}{ Mechanical ventilator settings during tracheostomy } \\
\hline Positive end expiratory pressure $\left(\mathrm{cm} \mathrm{H}_{2} \mathrm{O}\right)$ & $12(10-14)$ \\
\hline Peak inspiratory pressure $\left(\mathrm{cm} \mathrm{H}_{2} \mathrm{O}\right)$ & $15(12-16)$ \\
\hline Fraction of inspired oxygen & $0.60\left(0.35^{b}-0.80\right)$ \\
\hline Extracorporeal membrane oxygenation & $2(28.6)$ \\
\hline Continuous renal replacement therapy & $3(42.9)$ \\
\hline \multicolumn{2}{|l|}{ Laboratory data } \\
\hline Platelet count $\left(10^{3} / \mu l\right)$ & $159(82-196)$ \\
\hline Prothrombin time (sec) & $14.6(13.9-15.4)$ \\
\hline $\begin{array}{l}\text { Prothrombin time and international normalized } \\
\text { ratio }\end{array}$ & $1.17(1.06-1.25)$ \\
\hline Activated partial thromboplastin time (sec) & $37.5(36.2-46.4)$ \\
\hline \multicolumn{2}{|l|}{ Complications after tracheostomy } \\
\hline Bleeding & $2(28.6)$ \\
\hline
\end{tabular}

Values are presented as median (interquartile range) or number (\%). APACHE: Acute Physiology and Chronic Health Evaluation.

The duration from skin incision to insertion of the tracheostomy tube; betting during extracorporeal membrane oxygenation.

one required surgical exploration and transfusion of red blood cells and platelets. Of seven patients who underwent tracheostomy, the 30 -day mortality rate was 0 . However, by the end of the study, the mortality rate was $71.4 \%$ and no patients had the ventilator completely removed. Real-time RT-PCR results for SARS-CoV-2 in the upper and lower respiratory tract were positive in five cases (71.4\%) and negative in two cases (28.6\%). We evaluated viral load by calculating $\mathrm{Ct}$ values. The median Ct value for SARS-CoV-2 RdRp was 30.60 (IQR, 25.50-36.56) in the upper respiratory tract and 35.04 (IQR, 28.40-36.74) in the lower respiratory tract. In anti-SARS-CoV-2 antibodies tests, IgG and IgM were positive in all cases.

All tracheostomy procedures were performed in the negative pressure unit. However, four cases underwent tracheostomy in negative pressure cohort areas and three cases underwent tracheostomy in negative pressure isolation rooms. The COVID-19 ICU remained open for 80 days. After this unit closed, all medical staff, including the tracheostomy team, were tested for SARS-CoV-2 by real-time RT-PCR. All staff tested negative.

\section{DISCUSSION}

During treatment of patients with ARDS caused by COVID-19, aerosol-generating procedures carry the risk of infection transmission to medical personnel. Tracheostomy is an aerosolgenerating procedure commonly performed in critically ill patients. Several guidelines for tracheostomy in COVID-19 patients have been published to reduce the risk of infection transmission [7,8,13-15]. In terms of the environment, several reports $[7,8,13]$ demonstrate that standard personal protective equipment is essential when performing tracheostomy. No healthcare personnel became infected while performing tracheostomy during the SARS outbreak and all staff used a PAPR $[7,13]$. Tracheostomy is performed in a negative pressure environment and is best conducted at the bedside in an ICU $[7,8]$. In the current study, our medical staff wore a PAPR, a high durability hood with integrated head suspension, eye protection, a fluid-repellent disposable surgical gown, a cap, boots, double gloves, and an N95 mask as standard personal protective equipment. Such equipment is heavy and makes it difficult for healthcare personnel to tolerate loud noise and heat, but it protects them. The tracheostomy procedure was performed at the bedside in an ICU where negative pressure was maintained, although some procedures were performed in a negative pressure cohort area, not in a negative pressure isolation room.

In terms of the procedure, exposure to aerosolized secretions must be minimized during tracheostomy. McGrath et al. [8] reported that surgical tracheostomy was favored over percutaneous tracheostomy during the SARS outbreak. However, Zhang et al. [18] reported that percutaneous tracheostomy is preferable because it is minimally invasive and less time-con- 
suming, causes less splashing, and can be performed at the bedside, while viral splashing is more of a concern during surgical tracheostomy. Several suggestions to reduce viral aerosol spreading during tracheostomy have been published [18-21]. Various reports recommend that a muscle relaxant be used to eliminate the cough reflex during tracheostomy, the ETT cuff is placed deeper than the tracheal incision site, the ventilator is briefly paused while the tracheostomy tube is inserted, and the tracheal puncture site is covered with gauze $[8,13,18,20]$. There have also been various modified techniques using percutaneous tracheostomy [19-21]. However, we performed percutaneous tracheostomy in the same way as in patients without COVID-19. We just used a muscle relaxant to eliminate the cough reflex, and inserted the tracheostomy tube and balloon relatively quickly to reduce the amount of time as much as possible during which the virus could be transmitted. In addition, the tracheostomy site was covered with gauze and the operator's hands while the dilator was inserted and removed and when the tracheostomy tube was inserted.

A study found that the mean procedure time was 11 minutes for dilated tracheostomy and 14 minutes for surgical tracheostomy, with no statistical difference [22]. In another study, the mean duration of tracheostomy with Ciaglia Blue Rhino was 7.5 minutes [17]. In our study, the median procedure time was 10 minutes, which is similar to [22] or slightly longer than other studies [17]. Studies published up to the early 2000s showed that surgical tracheostomy was better for complications [22,23], but later studies showed that there was little difference in complications between surgical and dilated tracheostomy even in ECMO environments [24-26]. Regarding complications, in our study bleeding did not occur immediately after tracheostomy but developed within 24 hours after the procedure in two cases (28.6\%), both of which were on ECMO support and anticoagulation therapy. One case (14.3\%) required blood transfusion, and the other (14.3\%) was minor bleeding. A study [26] reported that dilated tracheostomy patients had $11 \%$ major bleeding requiring blood transfusion and $25 \%$ had minor bleeding in the ECMO environment. Therefore, compared with that study [26], our complication rate was similar.

In terms of viruses, although the procedure was not been modified, the viral load might be considered as one of the reasons healthcare personnel were not infected with COVID-19. Guidelines in the USA and Canada strongly advise that patients should test negative for COVID-19 before tracheostomy is performed $[14,15]$. However, it is uncertain whether a patient's condition will be maintained until they test negative for COVID-19 by RT-PCR. A study [5] reported that SARS-CoV-2 could be detected 2-3 weeks after symptoms developed and were identified for up to 37 days. Instead of waiting for patients to test negative by RT-PCR, we assumed that viral load and infectivity would be low if the $\mathrm{Ct}$ value was high. There is no clear standard regarding how the $\mathrm{Ct}$ value correlates with viral load. The Ct value cannot be used to directly interpret viral load without a standard curve generated using reference materials [27]. In addition, the sensitivity of qualitative RT-PCR is only $71 \%$ [28] and consequently false negative results must be sufficiently considered. The $\mathrm{Ct}$ value may provide more information about viral load. In our institution, a Ct value higher than 35 was considered a negative result. The median $\mathrm{Ct}$ values for SARS-CoV-2 RdRp were 30.60 in the upper respiratory tract and 35.04 in the lower respiratory tract, which are relatively high. Although viral load was not predicted correctly, it was considered to be low and this was thought to explain the low viral spread. Percutaneous tracheostomy was performed a median of 20 days after symptom onset and 14 days after application of the ventilator to reduce viral load. Early tracheostomy is thought to be unfavorable in patients with COVID-19.

This study evaluated the safety of percutaneous tracheostomy performed in a negative pressure environment in an ICU. We show that percutaneous tracheostomy performed in a negative pressure environment where some patients have COVID-19 is safe for medical staff and patients. This is thought to be because medical staff wore proper protection including a PAPR, the procedure was performed a median of 20 days after symptom onset and 14 days after application of the ventilator, and viral load was low. We believe that the risk of infection transmission by tracheostomy is related to the degree of healthcare personnel protection (including a PAPR), environmental factors such as a negative pressure environment, and even viral load (negative conversion may not be necessary), rather than viral shedding during the procedure.

Our study is limited because it only included seven cases and was retrospective, and it is difficult to apply in general because the results can vary depending on the condition of the operator and the patient. Further research is necessary. Dilated tracheostomy needs to be compared with surgical tracheostomy in the COVID-19 environment and non-COVID-19 tracheostomy results. It is necessary to do a prospective, multicenter study that includes more cases in the future.

\section{CONFLICT OF INTEREST}

No potential conflict of interest relevant to this article was reported. 


\section{ORCID}

$\begin{array}{ll}\text { Eun Jin Kim } & \text { https://orcid.org/0000-0001-9791-8077 } \\ \text { Eun-Hyung Yoo } & \text { https://orcid.org/0000-0002-6854-8932 } \\ \text { Chi Young Jung } & \text { https://orcid.org/0000-0002-8958-0886 } \\ \text { Kyung Chan Kim } & \text { https://orcid.org/0000-0001-5697-9674 }\end{array}$

\section{AUTHOR CONTRIBUTIONS}

Conceptualization: EJK. Data curation, Formal analysis, \& Methodology: EJK, EHY. Project administration: EJK. Visualization \& Writing-original draft: EJK, EHY. Writing-review \& editing: all authors.

\section{REFERENCES}

1. Huang C, Wang Y, Li X, Ren L, Zhao J, Hu Y, et al. Clinical features of patients infected with 2019 novel coronavirus in $\mathrm{Wu}$ han, China. Lancet 2020;395:497-506.

2. Chen N, Zhou M, Dong X, Qu J, Gong F, Han Y, et al. Epidemiological and clinical characteristics of 99 cases of 2019 novel coronavirus pneumonia in Wuhan, China: a descriptive study. Lancet 2020;395:507-13.

3. Wang D, Hu B, Hu C, Zhu F, Liu X, Zhang J, et al. Clinical characteristics of 138 hospitalized patients with 2019 novel coronavirus-infected pneumonia in Wuhan, China. JAMA 2020; 323:1061-9.

4. Guan WJ, Ni ZY, Hu Y, Liang WH, Ou CQ, He JX, et al. Clinical characteristics of coronavirus disease 2019 in China. N Engl J Med 2020;382:1708-20.

5. Zhou F, Yu T, Du R, Fan G, Liu Y, Liu Z, et al. Clinical course and risk factors for mortality of adult inpatients with COVID-19 in Wuhan, China: a retrospective cohort study. Lancet 2020; 395:1054-62.

6. Li X, Ma X. Acute respiratory failure in COVID-19: is it "typical" ARDS? Crit Care 2020;24:198.

7. Tay JK, Khoo ML, Loh WS. Surgical considerations for tracheostomy during the COVID-19 pandemic: lessons learned from the severe acute respiratory syndrome outbreak. JAMA Otolaryngol Head Neck Surg 2020;146:517-8.

8. McGrath BA, Brenner MJ, Warrillow SJ, Pandian V, Arora A, Cameron TS, et al. Tracheostomy in the COVID-19 era: global and multidisciplinary guidance. Lancet Respir Med 2020;8: 717-25.

9. Ahmed N, Hare GM, Merkley J, Devlin R, Baker A. Open tracheostomy in a suspect severe acute respiratory syndrome (SARS) patient: brief technical communication. Can J Surg
2005;48:68-71.

10. Kwan A, Fok WG, Law KI, Lam SH. Tracheostomy in a patient with severe acute respiratory syndrome. Br J Anaesth 2004;92: 280-2.

11. Wei WI, Tuen HH, Ng RW, Lam LK. Safe tracheostomy for patients with severe acute respiratory syndrome. Laryngoscope 2003;113:1777-9.

12. Song JH, Kim HC, Kang JG, Roh DH. Tracheotomy in Middle East respiratory syndrome: report of a case. J Clin Otolaryngol 2015;26:301-6.

13. Takhar A, Walker A, Tricklebank S, Wyncoll D, Hart N, Jacob $\mathrm{T}$, et al. Recommendation of a practical guideline for safe tracheostomy during the COVID-19 pandemic. Eur Arch Otorhinolaryngol 2020;277:2173-84.

14. Parker NP, Schiff BA, Fritz MA, Rapoport SK, Schild S, Altman $\mathrm{KW}$, et al. AAO position statement: tracheotomy recommendations during the COVID-19 pandemic [Internet]. Alexandria (VA): American Academy of Otolaryngology-Head and Neck Surgery; 2020 [cited 2020 Oct 15]. Available from: https: //www.entnet.org/content/aao-position-statement-tracheotomy-recommendations-during-covid-19-pandemic.

15. Engels PT, Weitzel E, Witterick IJ, Khalili S, Corsten M, Tewfik MA, et al. Recommendations from the CSO-HNS taskforce on performance of tracheotomy during the COVID-19 pandemic [Internet]. Elora (ON): Canadian Society of Otolaryngology-Head and Neck Surgery; 2020 [cited 2020 Oct 15]. Available from: https://www.entcanada.org/wp-content/uploads/ COVID-19-Guidelines-CSOHNS-Task-Force-Mar-23-2020.pdf.

16. Lee SY, Choi SH, Park JE, Hwang S, Kwon KT. Crucial role of temporary airborne infection isolation rooms in an intensive care unit: containing the COVID-19 outbreak in South Korea. Crit Care 2020;24:238.

17. Ambesh SP, Pandey CK, Srivastava S, Agarwal A, Singh DK. Percutaneous tracheostomy with single dilatation technique: a prospective, randomized comparison of Ciaglia blue rhino versus Griggs' guidewire dilating forceps. Anesth Analg 2002; 95:1739-45.

18. Zhang X, Huang Q, Niu X, Zhou T, Xie Z, Zhong Y, et al. Safe and effective management of tracheostomy in COVID-19 patients. Head Neck 2020;42:1374-81.

19. Angel L, Kon ZN, Chang SH, Rafeq S, Palasamudram Shekar S, Mitzman B, et al. Novel percutaneous tracheostomy for critically ill patients with COVID-19. Ann Thorac Surg 2020;110: 1006-11.

20. Vargas M, Russo G, Iacovazzo C, Servillo G. Modified percutaneous tracheostomy in COVID-19 critically ill patients. Head Neck 2020;42:1363-6. 
21. Al Yaghchi C, Ferguson C, Sandhu G. Percutaneous tracheostomy in patients with COVID-19: sealing the bronchoscope with an in-line suction sheath. Br J Anaesth 2020;125:e185-6.

22. Heikkinen M, Aarnio P, Hannukainen J. Percutaneous dilational tracheostomy or conventional surgical tracheostomy? Crit Care Med 2000;28:1399-402.

23. Dulguerov P, Gysin C, Perneger TV, Chevrolet JC. Percutaneous or surgical tracheostomy: a meta-analysis. Crit Care Med 1999;27:1617-25.

24. Pauliny M, Christova E, Mackova J, Liska M. Percutaneous dilation tracheostomy versus surgical tracheostomy in critically ill patients. Bratisl Lek Listy 2012;113:409-11.

25. Salna M, Tipograf Y, Liou P, Chicotka S, Biscotti M 3rd, Ager- strand C, et al. tracheostomy is safe during extracorporeal membrane oxygenation support. ASAIO J 2020;66:652-6.

26. Dimopoulos S, Joyce H, Camporota L, Glover G, Ioannou N, Langrish CJ, et al. Safety of percutaneous dilatational tracheostomy during veno-venous extracorporeal membrane oxygenation support in adults with severe respiratory failure. Crit Care Med 2019;47:e81-8.

27. Han MS, Byun JH, Cho Y, Rim JH. RT-PCR for SARS-CoV-2: quantitative versus qualitative. Lancet Infect Dis 2020 May 20 [Epub]. https://doi.org/10.1016/S1473-3099(20)30424-2.

28. Fang Y, Zhang H, Xie J, Lin M, Ying L, Pang P, et al. Sensitivity of chest CT for COVID-19: comparison to RT-PCR. Radiology 2020;296:E115-7. 